\title{
Déclin et survie des mobilités automobiles ? Entre résistances et évolutions
}

Sylvanie Godillon

Gaële Lesteven

Godillon, S. \& Lesteven, G. (2020). Déclin et survie des mobilités automobiles ? Entre résistances et évolutions. Flux, 119-120, 1-4. https://doi.org/10.3917/flux1.119.0001

Ce numéro-double de la Revue Flux se penche sur les mobilités automobiles. Il s'intéresse aux ruptures et aux continuités du système automobile mis en place au cours du siècle dernier, considérant l'évolution tant des usages que des politiques publiques.

\section{L'automobile au cour d'un contexte en évolution}

En septembre 2018, nous proposions un appel à articles sur les mobilités automobiles dans les espaces métropolitains, près de soixante ans après la parution de Déclin et survie des grandes villes américaines (Jacobs, 1961), ouvrage majeur remettant en cause la planification urbaine centrée sur l'automobile. En écho à Jane Jacobs, qui s'interrogeait sur la façon dont le trafic automobile érodait la ville et son urbanité, nous souhaitions questionner l'évolution du système automobile face aux évolutions technologiques et sociétales et aux préoccupations environnementales. L'objectif n'était pas de statuer sur un déclin des mobilités automobiles dont on sait qu'en dépit d'une stagnation des kilomètres parcourus observée au tournant des années 2000 (Goodwin, 2012), elles représentent toujours la majorité des déplacements quotidiens dans de nombreuses métropoles européennes (Focas, Christidis, 2017) et renforcent les inégalités socio-spatiales (Fol, 2009). Néanmoins, observe-t-on une évolution des pratiques de mobilité, pouvant entraîner à terme un changement de paradigme ou au contraire un renforcement du système automobile?

Nous cherchions, par cet appel, à éclairer les questionnements en lien avec les usages automobiles, les externalités qui en découlent et les politiques publiques qui les traitent, dans des contextes métropolitains où, au Nord comme au Sud, de plus en plus d'initiatives viennent du secteur privé, rendant plus complexe la gouvernance des mobilités automobiles.

Depuis le lancement de l'appel il y a deux ans, le contexte dans lequel s'inscrit cette réflexion a été bouleversé en France par plusieurs événements : l'approbation de la Loi d'Orientation des Mobilités (LOM) ; la baisse des vitesses maximales à $80 \mathrm{~km} / \mathrm{h}$ sur le réseau routier national, relevée depuis par Gilets jaunes tirant son origine dans des appels à manifester contre l'augmentation du prix des carburants, ou celui contre les réformes des retraites dont les grèves ont ralenti, voire mis à l'arrêt les transports en commun ; ou encore la pandémie du Covid19 provoquant un ralentissement historique des déplacements motorisés. Ces événements ont davantage rappelé que révélé l'ambivalence de la place de la voiture dans nos sociétés. Ainsi, la LOM a reconnu le rôle prépondérant de l'automobile dans les déplacements du quotidien 
tout en incitant au report modal vers les transports en commun et le vélo pour des questions environnementales; selon les Gilets jaunes, la nécessité d'utiliser l'automobile dans les territoires périurbains et ruraux représente des coûts démesurés; les grèves massives ont renforcé l'usage de la voiture, augmentant la congestion quand les transports en commun étaient à l'arrêt ; la pandémie du Covid19 a suscité des manières de « faire autrement » (notamment de ne pas se déplacer) alors que les modes de transport individuel permettent de garantir la distanciation interpersonnelle.

In fine, l'accélération des événements ne fait que renforcer le système automobile, qui profite de sa place prépondérante acquise au cours du siècle dernier et s'adapte efficacement aux évolutions contextuelles. Les articles de ce numéro-double sur les mobilités automobiles s'accordent pour souligner la place ambivalente de la voiture dans nos usages et nos politiques.

\section{Des usages aux politiques : l'étude des différentes faces du système automobile}

Les douze contributions de ce numéro-double, composé d'onze articles et d'un entretien croisé, illustrent la diversité des disciplines traitant du système automobile :de la sociologie à la géographie, en passant par l'histoire, l'aménagement, la science politique, l'informatique, le droit. Les méthodes mobilisées sont diverses (entretiens, carnets de bord embarqués dans les véhicules, statistiques, modélisation, analyse documentaire), tout comme les terrains d'études : de l'Europe francophone (France, Belgique et Suisse) à l'Amérique du Nord (Québec) et à l’Afrique (Sénégal).

Les articles de ce numéro empruntent deux directions principales : l'une sur les évolutions des usages automobiles et des valeurs associées ; l'autre sur l'aménagement, l'organisation et la planification de la mobilité et des territoires.

Les cinq premiers articles, complétés par un entretien sur le covoiturage, traitent des mobilités automobiles sous l'angle des usages. Prérequis à l'équipement automobile, le permis de conduire reste un rituel important du passage à l'âge adulte : Patrick Rérat et Lucas Haldimann montrent que la baisse constatée du taux de permis de conduire chez les jeunes Suisses s'explique davantage par un report de l'âge auquel il est passé que par un renoncement. Une fois équipés, les automobilistes ancrent leurs pratiques dans des routines, qui persistent malgré les perturbations du système automobile. À partir de carnets de bords et de parcours commentés, Joël Meissonnier et Cyprien Richer constatent que l'allongement des temps de parcours dû à la congestion n'est pas forcément vécu négativement par les automobilistes, devenant même pour certains un refuge pour décompresser. La hausse des préoccupations environnementales ne remet pas plus en cause l'automobile, pourtant l'un des principaux producteurs d'externalités négatives. Selon Yoann Demoli, Matéo Sorin et Axel Villareal, le recours à la voiture et l'intensité de son usage s'expliquent essentiellement par des facteurs contextuels, liés à la densité du lieu de résidence et aux positions dans le cycle de vie et dans l'échelle des revenus, les valeurs environnementales n'influant qu'à la marge. En analysant les processus de démotorisation des ménages, Leslie Belton Chevallier et Joseph Cacciari soulignent à quel point le renoncement à l'automobile peut être un facteur de stigmatisation pour les individus ou les ménages. Le texte de Lourdes Diaz Olvera, Didier Plat et Pascal Pochet propose une lecture 
différente des usages automobiles à partir du traitement d'enquêtes réalisées à Dakar. Dans la capitale sénégalaise, la voiture reste un bien rare, essentiellement détenu par les catégories sociales aisées. Mais son usage se diffuse au-delà du cercle des citadins pouvant en conduire une de façon permanente. L'usage partagé de la voiture à Dakar fait écho aux initiatives de covoiturage dans les pays du Nord. Dans un entretien croisé, Jean-Baptiste Ray montre que le covoiturage constitue un potentiel de mobilité local important et participe au renouvellement des usages de la voiture, tandis qu'Éléonore Pigalle appelle à avoir un regard critique sur la promotion du covoiturage.

La seconde moitié des articles de ce numéro se regroupent autour du thème de l'aménagement, de la planification et des politiques de mobilité. Par une analyse rétrospective, Frédéric Héran discute l'usage du terme de « tout automobile », consistant à accorder la priorité à la voiture sur tous les autres modes de déplacement en toutes circonstances. La place de la voiture est au cœur des politiques de mobilité dans les grandes villes des pays du Nord. En comparant les politiques menées à Bruxelles et à Paris, Jean Debrie, Juliette Maulat et Sandrine Berroir concluent sur une standardisation des politiques alternatives à l'automobile avec de fortes incitations au report modal mais peu de contraintes pour limiter l'usage de la voiture. Ces enjeux de gouvernance se retrouvent à l'échelle locale. Étudiant le développement de quartiers de gare dans la région de Montréal, Florence Paulhiac-Scherrer et Blandine Vernier révèlent comment la gestion du stationnement pose un défi de coordination entre acteurs du transport et de l'urbanisme, mais également avec les promoteurs et la population. Plus largement, Jérôme Laviolette, Catherine Morency et Owen Waygood font le constat qu'au Québec, les mobilités automobiles continuent à croître, les documents de planification et les projets d'infrastructure ne vont pas dans le sens d'une réduction de l'usage de l'automobile, malgré une reconnaissance de la dépendance à l'automobile et des enjeux associés, notamment environnementaux. Le cas français fait écho à ce constat. Patricia Sajous, Paul Salze et Valérie Bailly-Hascoët montrent que si le «paysage » réglementaire est en place pour un moindre usage de la voiture, l'automobile demeure centrale dans les usages comme dans les politiques mises en œuvre. Le système automobile se transforme alors pour faire face aux injonctions de durabilité. Concluant ce numéro, l'essai de Gabriel Dupuy rappelle comment la voiture est devenue indispensable à la vie dans des territoires que les automobilistes ont peu à peu redessinés (Dupuy, 1995). Or, selon son analyse, les solutions qui permettraient de sortir de la dépendance en redéfinissant des territoires sans automobile mènent à des impasses. C'est donc l'automobile elle-même qu'il faut mettre en cause en s'appuyant, par exemple, sur le développement du véhicule autonome.

\section{Les apports du numéro}

Les articles de ce numéro s'accordent sur deux apports principaux. D'une part, les usages de l'automobile restent socialement et spatialement différenciés. D'autre part, seule une action publique ambitieuse permet de reconsidérer la place de l'automobile. 


\section{Des usages socialement et spatialement différenciés}

La possession et l'usage d'une voiture permettent à l'individu de s'inscrire dans la norme dominante de l'automobilité (Urry, 2004). Cependant, les mobilités automobiles restent caractérisées par d'importantes différences de genre : les jeunes femmes sont plus nombreuses à ne pas avoir de permis de conduire (Rérat et Haldimann), à avoir « peur » de conduire (Belton Chevallier et Cacciari) et à avoir moins accès à l'automobile (Diaz Olvera, Plat et Pochet). Cette différence de genre se couple d'une différence de classe sociale. Les jeunes issus de familles dans laquelle la situation financière est jugée bonne ont une plus grande probabilité de détenir le permis de conduire (Rérat et Haldimann); les ménages non motorisés sont souvent plus pauvres que les ménages motorisés (Demoli, Sorin et Villareal). Les lignes se brouillent quand intervient le capital culturel. Lorsqu'il est élevé, la valorisation de la voiture est moindre (Rérat et Haldimann). Une forte valorisation de la voiture est prégnante parmi les classes populaires en Europe comme en Afrique (Diaz Olvera, Plat et Pochet). En France, le permis de conduite et l'accès à la voiture revêtent une fonction assurantielle, en particulier chez les ménages populaires qui résident en dehors des centres urbains (Rérat et Haldimann ; Demoli, Sorin et Villareal; Belton Chevallier et Cacciari), et que confortent les routines du quotidien (Meissonnier et Richer). Cette socialisation longue et précoce à l'automobile en rend l'équipement impensé et le non-équipement impensable (Demoli, Sorin et Villareal) ; stigmatisant ceux qui n'en possèdent plus (Belton Chevallier et Cacciari).

Les différences territoriales demeurent très marquées, notamment entre les villes centres et les territoires périurbains ou ruraux. L'étalement urbain renforce la dépendance automobile alors que les alternatives à l'automobile dans les territoires moins denses font défaut (Dupuy ; Sajous, Salze et Bailly- Hascoët ; Meissonnier et Richer).

\section{L'action publique à l'épreuve du système automobile}

Après des décennies de politiques orientées vers l'automobile (Héran), l'hégémonie automobile apparaît en perte de vitesse, sous la montée des préoccupations environnementales tant du côté des usagers (Demoli, Sorin et Villareal) que des pouvoirs publics (Laviolette, Morency et Waygood). Toutefois, les pouvoirs publics continuent à penser leurs politiques de mobilité autour de l'automobile (Héran; Laviolette, Morency et Waygood ; Sajous, Salze et BaillyHascoët). Ils incitent à la requalification des espaces centraux et au report vers d'autres modes de transport sans contraindre l'usage automobile (Debrie, Maulat et Berroir ; Paulhiac Scherrer et Vernier). Face à ces politiques, les usagers s'adaptent, sans forcément renoncer à l'automobile (Meissonnier et Richer).

Les évolutions des mobilités automobiles s'inscrivent dans une continuité qui peut être qualifiée de dépendance au sentier (Briggs, Webb, Clevo, 2015) ou de chemin schizophrénique (Banister, 2008). Autrement dit, nous nous trouvons dans une situation où les externalités négatives sont identifiées ; les enjeux sont connus, notamment ceux en lien avec le réchauffement climatique ; les alternatives à l'automobile existent ; mais où le système continue à fonctionner comme il le fait depuis des décennies, sans réelles actions pour changer la situation. Les politiques 
urbaines qui cherchent à contraindre la voiture sont ambigües car dans le même temps elles confortent son rôle (Sajous, Salze et Bailly-Hascoët).

Pour en sortir, l'action publique a un rôle clé à jouer : à l'échelle des quartiers où une gestion concertée du stationnement peut le rendre plus durable (Paulhiac Scherrer et Vernier), à l'échelle métropolitaine où un portage politique ambitieux peut faire face aux résistances structurées des associations et des groupes d'intérêt automobilistes (Debrie, Maulat et Berroir ; Laviolette, Morency et Waygood), à l'échelle de la planète où les enjeux de la transition écologique sont à considérer dans leur ensemble (Héran). Une régulation effective des trafics automobiles n'est pérenne que si des alternatives de qualité à l'automobile existent, adaptées aux populations, aux échelles et aux territoires considérés.

Si aujourd'hui nous sommes dans une phase de « déclin » des politiques promouvant la voiture individuelle, nous demeurons dans une phase de « survie » des pratiques et des valeurs positives associées à ce mode de transport. Plus que jamais, il importe de questionner la place de l'automobile dans nos sociétés et nos territoires.

Sylvanie Godillon est géographe et urbaniste, spécialiste des enjeux de mobilité et des politiques urbaines. Ses travaux portent sur les externalités des transports (risque d'accident, bruit, pollution de l'air, impacts climatiques, etc.) et les politiques urbaines de mobilité (généralisation de la vitesse à 30 $\mathrm{km} / \mathrm{h}$, programme d'incitation au changement de mode, etc.). Elle est chercheuse associée à l'UMR Géographie-cités.

Sylvanie.godillon@gmail.com

Gaële Lesteven est géographe et urbaniste. Chargée de recherche à l'École nationale des Ponts et des Chaussées, au sein du Laboratoire Ville Mobilité Transport, elle étudie les systèmes de mobilité urbaine à l'échelle internationale. Ses travaux portent sur l'étude des innovations dans les pratiques et les services de mobilité et sur l'articulation entre politiques de transport et politiques d'aménagement.

Gaele.lesteven@enpc.fr

\section{Bibliographie}

Banister D., 2008, The sustainable mobility paradigm, Transport Policy, 15(2), p. 73-80.

Briggs M., Webb J., Clevo W., 2015, Automotive Modal Lockin: The role of path dependence and large socio-economic regimes in market failure, Economic Analysis and Policy, 45, p. 5868 .

Cacciari J., Leslie Belton Chevallier L., 2020, La démotorisation des ménages comme analyseur de la diversité des expériences de socialisation à la « norme automobile », Flux, 2020/1-2 ( ${ }^{\circ}$ 119-120), p. 59-72.

Debrie J., Maulat J., Berroir S., 2020, Les politiques urbaines face à l'automobile : objectifs, outils et controverses de l'action publique dans les métropoles de Bruxelles et Paris, Flux, 2020/1-2 ( $\left.\mathrm{N}^{\circ} 119-120\right)$, p. 102-120. 
Demoli Y., Matéo Sorin M., Villaereal A., 2020, Conversion écologique vs dépendance automobile. Une analyse des dissonances entre attitudes environnementales et usages de l'automobile auprès de ménages populaires en zone périurbaine et rurale, Flux, 2020/1-2 ( $\mathrm{N}^{\circ}$ 119-120), p. 41-58.

Diaz Olvera L., Plat D., Pochet P., 2020, Accéder à l'automobile dans les villes d'Afrique subsaharienne : usages et usagers dakarois, Flux, 2020/1-2 ( $\left.\mathrm{N}^{\circ} 119-120\right)$, p. 73-89.

Dupuy, G., 1995, Les territoires de l'automobile, Paris : Anthropos-Economica.

Dupuy G., 2020, Voiture autonome : la fin des territoires de l'automobile ?, Flux, 2020/1-2 ( ${ }^{\circ}$ 119-120), p. 185-191.

Focas C., Christidis P., 2017, What drives car use in Europe?, European Commission, Joint Research Centre.

Fol S., 2009, La mobilité des pauvres : pratiques d'habitants et politiques publiques, Paris : Belin.

Goodwin P., 2012, Peak Travel, Peak Car and the Future of Mobility: Evidence, Unresolved Issues, Policy Implications, and a Research Agenda, Discussion Paper, OECD.

Héran F., 2020, La remise en cause du tout automobile, Flux, 2020/1-2 ( $\left.\mathrm{N}^{\circ} 119-120\right)$, p. 90101.

Jacobs J., 1961, The Death and Life of Great American Cities, New York: Vintage.

Laviolette J., Morency, Waygood C. O., 2020, Persistance de l'automobilité ? Analyse en trois perspectives, Flux, 2020/1-2 (N 119-120), p. 142-172.

Meissonnier J., Richer C., 2020, Les routines automobiles à l'épreuve des perturbations. Comprendre les résistances au changement à partir de récits d'usagers dans la métropole lilloise, Flux, 2020/1-2 (N¹19-120), p. 25-40.

Paulhiac Scherrer F., Vernier B., 2020, L'émergence du stationnement-durable dans les TOD de banlieue. Regards croisés sur deux projets de la région montréalaise, Flux, 2020/1-2 ( ${ }^{\circ}$ 119-120), p. 121-141.

Rérat P., Haldimann L., 2020, Le permis de conduire chez les jeunes : qui ne le passe pas (encore) et pourquoi ?, Flux, 2020/1-2 ( $\left.\mathrm{N}^{\circ} 119-120\right)$, p. 5-24.

Sajous P., Salze P., Bailly-Hascoët V., 2020, Système automobile et modèles de transports : quelles évolutions pour planifier la mobilité de demain ?, Flux, 2020/1-2 ( $\left.\mathrm{N}^{\circ} 119-120\right)$, p. 173184.

Urry J., 2004, The 'System' of Automobility, Theory, Culture \& Society, 21(4-5), p. 25-39.

Entretien, Flux, 2020/1-2 (ํ119-120), p. 192-196. 